
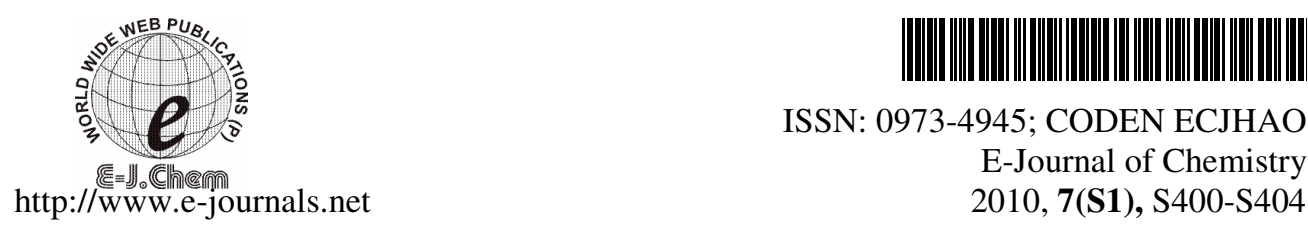

ISSN: 0973-4945; CODEN ECJHAO

E-Journal of Chemistry

2010, 7(S1), S400-S404

\title{
Synthesis and Antimicrobial Activity of Some Aldehyde Derivatives of 3-Acetylchromen-2-one
}

\author{
B.LAKSHMINARAYANAN", V.RAJAMANICKAM, \\ T.SUBBURAJU, L.A. PRADEEP RAJKUMAR and H.REVATHI ${ }^{\S}$ \\ *Karpagam College of Pharmacy \\ S.F.No:762, Othakkalmandapam, Coimbatore- 641032, India \\ Arulmigu Kalasalingam College of Pharmacy \\ Anand Nagar, Krishnankoil- 626190, India \\ ${ }^{\S}$ Wockhardt Limited, Biotech Park \\ H-14/2 m.i.d.c. Area, Waluj, Aurangabad- 431136, India \\ arunrxpharma@yahoo.co.in
}

Received 11 March 2010; Accepted 5 May 2010

\begin{abstract}
Some new 3-(substituted)-chromen-2-one have been synthesized by condensation of 3-acetylchromen-2-one with various aromatic aldehyde in presence of ethanol and alkali. The synthesized compounds were identified by spectral data and screened for their antibacterial activity against $B$. pumilis, $B$. substilis and E. coli and antifungal activity against A. niger and Candida albicans. Among the synthesized compounds, some compounds of aryl chromen, which are having electron releasing substituent such as methoxy and hydroxyl at various positions, showed moderate to considerable antibacterial and antifungal activities.
\end{abstract}

Keywords: Chromen, Synthesis, Antibacterial activity, Antifungal activity.

\section{Introduction}

Chromen moiety display interesting biological activities including anti-inflammatory ${ }^{1}$, analgesic $^{2}$, antifungal ${ }^{3}$, antibacterial ${ }^{4}$, antiviral ${ }^{5}$, antipsychotic ${ }^{6}$, antituberculosis ${ }^{7}$, anti-HIV ${ }^{8}$, and anticarcinogenic ${ }^{9}$ activities. In the present study, we synthesized different 3(substituted)-chromen-2-one derivatives by condensing 3 -acetylchromen-2-one ${ }^{10}$ with different aromatic aldehyde in presence of alcohol and alkali. The structures of the various synthesized compounds are assigned on the basis of elemental analysis and spectral studies $^{11,12}$. These compounds were also screened for antimicrobial activity.

\section{Experimental}

Melting points were determined by open ended capillary tube in the electrical melting point apparatus and are uncorrected and the purity of the compounds were checked by TLC using 
silica gel as stationary phase and the spots were visually detected in an iodine chamber. The structures of the compounds were elucidated by IR (Perkin-Elmer) in $\mathrm{KBr}$ disc and ${ }^{1} \mathrm{H}$ NMR (Bruker Spectrospin AV $400 \mathrm{MHz}$ Spectrometer using TMS as an internal standard).

\section{General procedure for the preparation of chromens (a-f)}

A mixture of 3 -acetylchromen-2-one ${ }^{10}(0.0304 \mathrm{~mol})$ and appropriate aldehyde $(0.0304 \mathrm{~mol})$ was stirred in water $(40 \mathrm{~mL})$ and ethanol $(25 \mathrm{~mL})$ in presence of sodium hydroxide $(0.0302 \mathrm{~mol})$ for $5 \mathrm{~h}$. The reaction mixture was kept overnight in an ice bath. Then the above mixture was poured into a beaker containing water. The precipitated product was filtered and recrystallized from ethanol (Scheme 1). The characterization data of these compounds are described in Table $1 \& 2$.<smiles>[R]C=O</smiles>

3-acetylchromen-2-one
Aromatic aldehyde<smiles>O=C(/C=C/P)c1cc2ccccc2oc1=O</smiles>

Compounds (a-f) b<smiles>COc1cc(C)ccc1O</smiles>

e<smiles>COc1cc(C)cc(OC)c1OC</smiles>

c<smiles>Cc1ccc(C)c(Cl)c1</smiles>

f

Scheme 1. Synthesis of some new 3-(substituted)-chromen-2-one (a-f)

Table 1. Characterization data of the compounds (a-f)

\begin{tabular}{|c|c|c|c|c|c|c|c|c|c|}
\hline \multirow{3}{*}{ Compd. } & \multirow{3}{*}{$\begin{array}{l}\text { Molecular } \\
\text { Formula }\end{array}$} & \multirow{3}{*}{${ }^{\mathrm{m}} \mathrm{C}, \mathrm{C}$} & \multirow{3}{*}{$\begin{array}{c}\text { Yield, } \\
\%\end{array}$} & \multicolumn{6}{|c|}{ Elemental analysis, \% } \\
\hline & & & & \multicolumn{2}{|c|}{$\mathrm{C}$} & \multicolumn{2}{|c|}{$\mathrm{H}$} & \multicolumn{2}{|c|}{$\mathrm{O}$} \\
\hline & & & & Found & Calcd. & Found & Calcd. & Found & Calcd. \\
\hline $\mathbf{a}$ & $\mathrm{C}_{20} \mathrm{H}_{17} \mathrm{O}_{3} \mathrm{~N}$ & 66 & 78 & 89.42 & 89.56 & 5.90 & 5.94 & 10.12 & 10.23 \\
\hline b & $\mathrm{C}_{19} \mathrm{H}_{14} \mathrm{O}_{4}$ & 62 & 69 & 68.56 & 68.58 & 5.10 & 5.16 & 11.72 & 11.80 \\
\hline c & $\mathrm{C}_{21} \mathrm{H}_{18} \mathrm{O}_{6}$ & 72 & 88 & 85.18 & 85.38 & 4.63 & 4.88 & 10.23 & 10.36 \\
\hline d & $\mathrm{C}_{21} \mathrm{H}_{18} \mathrm{O}_{6}$ & 69 & 85 & 85.18 & 85.38 & 4.63 & 4.88 & 10.23 & 10.36 \\
\hline e & $\mathrm{C}_{19} \mathrm{H}_{14} \mathrm{O}_{5}$ & 71 & 74 & 66.45 & 66.58 & 3.28 & 3.21 & 9.98 & 9.79 \\
\hline f & $\mathrm{C}_{18} \mathrm{H}_{10} \mathrm{O}_{3} \mathrm{Cl}_{2}$ & 82 & 91 & 82.69 & 82.74 & 4.81 & 5.01 & 11.24 & 11.37 \\
\hline
\end{tabular}


Table 2. Spectral data of the compounds (a-f)

\begin{tabular}{|c|c|c|}
\hline Compd. & $\mathrm{IR}\left(\mathrm{KBr}, \mathrm{cm}^{-1}\right)$ & ${ }^{1} \mathrm{H} \mathrm{NMR}\left(\mathrm{CDCl}_{3}, \mathrm{ppm}\right)$ \\
\hline $\mathbf{a}$ & $\begin{array}{l}1725(-\mathrm{C}=\mathrm{O}), 1359.57(\mathrm{C}-\mathrm{N}, \text { stretch, } \\
\left.3^{0} \text { amine }\right), 1439.6\left(\mathrm{CH}_{3}\right), 1595.81 \\
(-\mathrm{C}=\mathrm{C}-), 1066.44,1231.33(-\mathrm{C}=\mathrm{C}-\mathrm{O}-\mathrm{C}-)\end{array}$ & $\begin{array}{l}2.206\left(\mathrm{~s}, 3 \mathrm{H}, \mathrm{CH}_{3}\right), 3.314\left(\mathrm{~s}, \mathrm{CH}_{3}\right) \\
6.645-7.12(\mathrm{~m}, \mathrm{Ar}-\mathrm{H}), 2.85-2.93 \\
\left(\mathrm{~s}, 6 \mathrm{H},-\mathrm{N}\left(\mathrm{CH}_{3}\right)_{2}\right)\end{array}$ \\
\hline b & $\begin{array}{l}1730(-\mathrm{C}=\mathrm{O}), 1447.31\left(-\mathrm{CH}_{3}\right) 1596.77 \\
(-\mathrm{C}=\mathrm{C}-), 1600(-\mathrm{C}=\mathrm{C}-) 2812.67\left(-\mathrm{O}-\mathrm{CH}_{3}\right)\end{array}$ & $\begin{array}{l}3.951\left(3 \mathrm{H},-\mathrm{O}-\mathrm{CH}_{3}\right), 6.712-8.085 \\
(\mathrm{~m}, \mathrm{Ar}-\mathrm{H}), 6.2\left(\mathrm{~d}, 1 \mathrm{H}, \mathrm{C}_{2} \mathrm{H}\right)\end{array}$ \\
\hline c & $\begin{array}{l}1735(-\mathrm{C}=\mathrm{O}), 1638(-\mathrm{CH}=\mathrm{CH}-), \\
1180\left(-\mathrm{O}-\mathrm{CH}_{3}\right), 1602(-\mathrm{C}=\mathrm{C}-)\end{array}$ & $\begin{array}{l}3.98\left(9 \mathrm{H}, \mathrm{s}, 3 \mathrm{xO}-\mathrm{CH}_{3}\right), 6.89(1 \mathrm{H}, \mathrm{d} \\
-\mathrm{CO}-\mathrm{CH}=), 7.73(1 \mathrm{H}, \mathrm{d},-\mathrm{CH}-\mathrm{Ar}) \\
7.41-7.82(4 \mathrm{H}, \mathrm{m}, \mathrm{Ar}-\mathrm{H})\end{array}$ \\
\hline d & $\begin{array}{l}1728(-\mathrm{C}=\mathrm{O}), 1642(-\mathrm{CH}=\mathrm{CH}-), 1182 \\
\left(-\mathrm{O}-\mathrm{CH}_{3}\right), 1622(-\mathrm{C}=\mathrm{C}-), 1185\left(-\mathrm{O}-\mathrm{CH}_{3}\right)\end{array}$ & $\begin{array}{l}3.78\left(3 \mathrm{H}, \mathrm{d}, \mathrm{O}-\mathrm{CH}_{3}\right), 6.85(1 \mathrm{H}, \mathrm{d}, \\
-\mathrm{CO}-\mathrm{CH}=), 7.43(1 \mathrm{H}, \mathrm{d},-\mathrm{CH}-\mathrm{Ar}) \\
7.56-7.95(4 \mathrm{H}, \mathrm{m}, \mathrm{Ar}-\mathrm{H})\end{array}$ \\
\hline e & $\begin{array}{l}1741(-\mathrm{C}=\mathrm{O}), 1635(-\mathrm{CH}=\mathrm{CH}-), \\
1178\left(-\mathrm{O}-\mathrm{CH}_{3}\right), 1612(-\mathrm{C}=\mathrm{C}-)\end{array}$ & $\begin{array}{l}3.89\left(3 \mathrm{H}, \mathrm{s}, 3 \mathrm{xO}-\mathrm{CH}_{3}\right), 6.98(1 \mathrm{H}, \mathrm{d}, \\
-\mathrm{CO}-\mathrm{CH}=), 7.78(1 \mathrm{H}, \mathrm{d},-\mathrm{CH}- \\
\mathrm{Ar}), 9.81(1 \mathrm{H}, \mathrm{s},-\mathrm{OH}), 6.78-7.02(7 \mathrm{H}, \mathrm{m}, \\
\mathrm{Ar}-\mathrm{H})\end{array}$ \\
\hline $\mathbf{f}$ & $\begin{array}{l}\text { 1728(-C=O), 1650(-CH=CH- }), \\
1610(-\mathrm{C}-\mathrm{Cl}), 1257.36(-\mathrm{C}=\mathrm{C}-\mathrm{O}-\mathrm{C}-)\end{array}$ & $\begin{array}{l}\text { 6.91(1H, d, -CO-CH=), 7.61(1H, d, - } \\
\text { CH-Ar }), 7.32-7.96(7 \mathrm{H}, \mathrm{m}, \mathrm{Ar}-\mathrm{H})\end{array}$ \\
\hline
\end{tabular}

\section{Results and Discussion}

Antimicrobial activity ${ }^{13}$

Cup plate method ${ }^{13-15}$ using Mueller-Hinton agar medium was employed to study the preliminary antibacterial activity of (a-f) against B.pumilis, B.substilis and E.coli. The agar medium was purchased from HiMedia Laboratories Ltd., Mumbai, India. Preparation of nutrient broth, subculture, base layer medium, agar medium and peptone water was done as per the standard procedure ${ }^{14-16}$. Each test compounds $(5 \mathrm{mg})$ was dissolved in $5 \mathrm{~mL}$ of dimethyl sulfoxide $(1000 \mu \mathrm{g} / \mathrm{mL})$. Volume of $0.05 \mathrm{~mL}$ and $0.1 \mathrm{~mL}$ of each compound were used for testing. gentamycin was used as a reference drug and dimethyl sulfoxide as a control, which did not revealed any inhibition.

Same cup plate method using Sabouraud Dextrose Agar (SDA) medium was employed to study the preliminary antifungal activity of (a-f) against A. niger and candida albicans. The SDA agar medium was purchased from HiMedia Laboratories Ltd., Mumbai, India. Preparation of nutrient broth, subculture, base layer medium, agar medium and peptone water was done as per the standard procedure ${ }^{14-16}$. Each test compounds $(5 \mathrm{mg})$ was dissolved in $5 \mathrm{~mL}$ of dimethyl sulfoxide $(1000 \mu \mathrm{g} / \mathrm{mL})$. Volume of $0.05 \mathrm{~mL}$ and $0.1 \mathrm{~mL}$ of each compound were used for testing. Griseofulvin was used as a reference drug and dimethyl sulfoxide as a control, which did not revealed any inhibition.

The cups each of $9 \mathrm{~mm}$ diameter were made by scooping out medium with a sterilized cork borer in a petri dish, which was streaked with the organisms. The solutions of each test compound $(0.05$ and $0.1 \mathrm{~mL})$ were added separately in the cups and petri dishes and were subsequently incubated. Zone of inhibition produced by each compound was measured in $\mathrm{mm}$ and the results are presented in Table 3 . 
Table 3. Zone of inhibition of compound (a-f)

\begin{tabular}{ccccccccccc}
\hline Compd. & \multicolumn{3}{c}{ B.pumilis } & \multicolumn{2}{c}{ B. substilis } & \multicolumn{2}{c}{ E.coli } & \multicolumn{2}{c}{ A.niger } & \multicolumn{2}{c}{ C.albicans } \\
\cline { 2 - 11 } No & 0.05 & 0.1 & 0.05 & 0.1 & 0.05 & 0.1 & 0.05 & 0.1 & 0.05 & 0.1 \\
& $\mathrm{~mL}$ & $\mathrm{~mL}$ & $\mathrm{~mL}$ & $\mathrm{~mL}$ & $\mathrm{~mL}$ & $\mathrm{~mL}$ & $\mathrm{~mL}$ & $\mathrm{~mL}$ & $\mathrm{~mL}$ & $\mathrm{~mL}$ \\
\hline $\mathbf{a}$ & 10 & 11 & -- & 05 & 10 & 12 & -- & 06 & 11 & 13 \\
b & 11 & 14 & 13 & 15 & 14 & 16 & 15 & 17 & 13 & 15 \\
$\mathbf{c}$ & 15 & 20 & 16 & 19 & 14 & 18 & 14 & 16 & 17 & 19 \\
$\mathbf{d}$ & 14 & 18 & 15 & 18 & 14 & 17 & 15 & 19 & 14 & 17 \\
$\mathbf{e}$ & 13 & 15 & 15 & 16 & 14 & 16 & 14 & 17 & 12 & 13 \\
$\mathbf{f}$ & -- & 05 & 06 & 10 & 10 & 12 & 08 & 10 & 12 & 13 \\
$\mathrm{GM}$ & 21 & 25 & 20 & 21 & 18 & 20 & $*$ & $*$ & $*$ & $*$ \\
$\mathrm{GF}$ & $*$ & $*$ & $*$ & $*$ & $*$ & $*$ & 19 & 20 & 20 & 23 \\
\hline
\end{tabular}

GM-Gentamycin, GF-Griseofulvin, (*) indicates activity not done, (--) indicates no zone of inhibition

\section{Conclusion}

All the newly synthesized compounds were structure elucidated by spectral data and screened for their antimicrobial activity. Among all the tested compounds (b), (c), (d) and (e) displayed considerable activity against all the pathogenic organisms.

From the above results, it is interesting to note that aryl chromen, which are having electron releasing substituent such as methoxy and hydroxyl at various positions, showed moderate to considerable antibacterial and antifungal activities. It also notes that increasing methoxy group, increases the activity.

\section{Acknowledgments}

The authors are thankful to Head, Department of Chemistry, Madurai Kamarajar University, Madurai (India) for providing ${ }^{1} \mathrm{H}$ NMR spectra. We are thankful to Head, Dept. of pharmaceutical analysis, SRIMPS, Coimbatore (India) for IR spectra. Author are grateful to Grace College of Pharmacy, Palakkad, Kerala (India) for providing the laboratory facilities and granting permission to carry out part of the research work.

\section{References}

1. Manjunath Ghate, Monohar V Kulkarni, Indian J Chem., 2005, 44B, 1674-1678.

2. Akhlaq Waheed and Suroor Ahmed Khan, Indian J Heterocycl Chem., 2001, 11, 59-62

3. Bharati P Choudhari and Mulwad V V, Indian J Chem., 2006, 45B, 309-313.

4. Mashooq A Bhat, Khan S A and Siddiqui N, Indian J Heterocycl Chem., 2005, 3, 271.

5. Pandey V K, Singh V K, Tandon M, Joshi M N and Bajpai S K, Indian J Chem, 2004, 43B, 1790

6. Shinde R M, Bhosale S H, Bhosale M S, Gawai A A, Mahadik K R and Kadam S S, Indian J Heterocycl Chem., 2006, 15(3), 267.

7. Rajeswar Rao V and Modan Mohan Reddy M, Indian J Heterocycl Chem., 2003, 13, 69-72.

8. Mausami Chavda, Denish Karia and Ahamik Shah, Indian J Chem., 2002, 41B, 2197.

9. Nair R V, Fisher E P, Safe S H, Cortez C, Harvey R G and DiGiovanni J, Carcinogenesis, 1991, 12(1), 65-69.

10. Mogilaiah K, Ch. Srinivas Reddy and Randheer Reddy G, Indian J Heterocycl Chem., 2002, 12, 285-286. 
11. Dyer J R, Applications of absorption spectroscopy or organic Compounds, $1^{\text {st }}$ Edn., 1996, 33-38.

12. Willard H H, Merritt L L, Dean J A and Settle F A, Instrumental Methods of Analysis, $7^{\text {th }}$ Edn., 1986, p.310.

13. Rajendra Prasad Y, Lakshmana Rao A and Rambabu R, E-J Chem., 2008, 5(3) 461-466.

14. Bowden K, Dal Pozzo A and Duah C K, J Chem Res (S), 1990, 377.

15. Seely $\mathrm{H} \mathrm{W}$ and Van Denmark P J, Microbs in Action: A Laboratory Manual of Microbiology, D B Taraporewala Sons and Co, Bombay, 1975, 55-80.

16. Miller J L, Clinical diagnosis and management by Laboratory methods, $17^{\text {th }}$ Edn., 2001, p.765. 


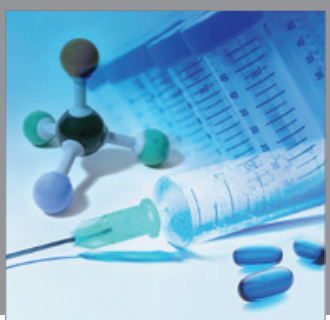

International Journal of

Medicinal Chemistry

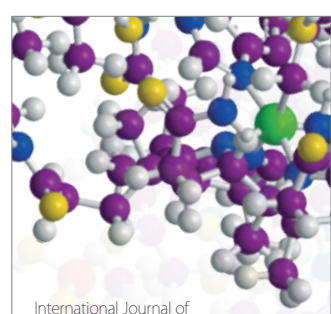

Carbohydrate Chemistry

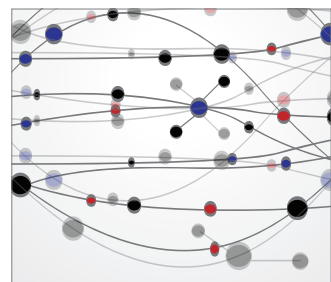

The Scientific World Journal
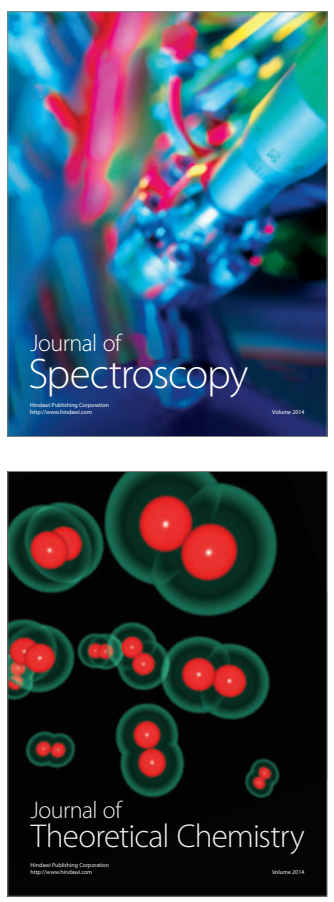
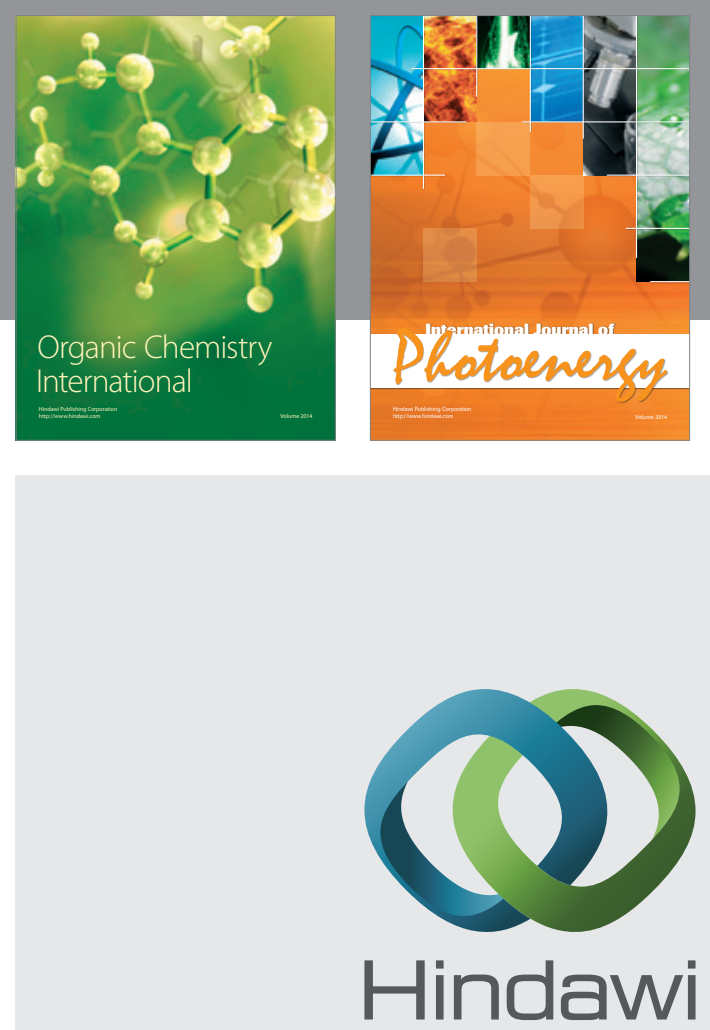

Submit your manuscripts at

http://www.hindawi.com
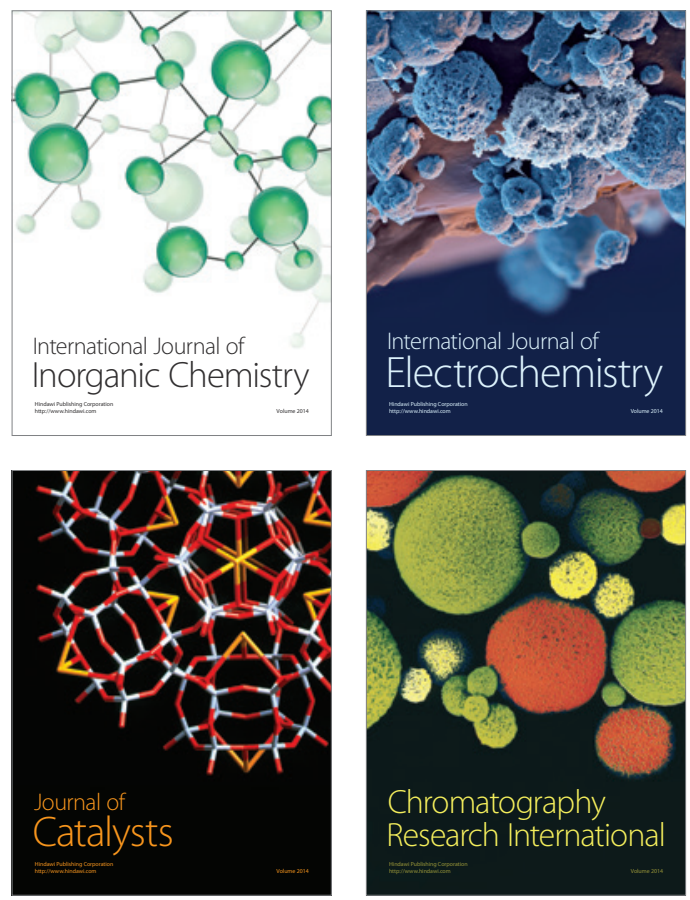
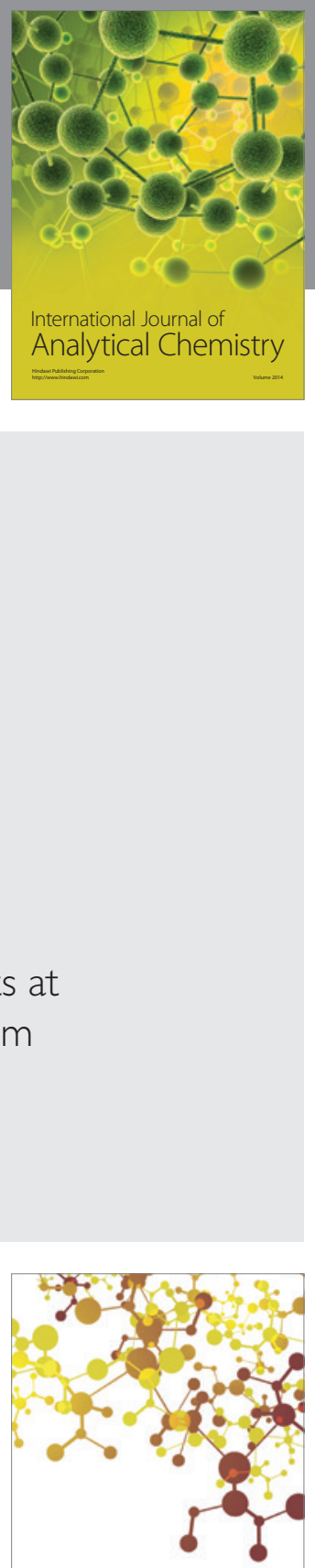

Journal of

Applied Chemistry
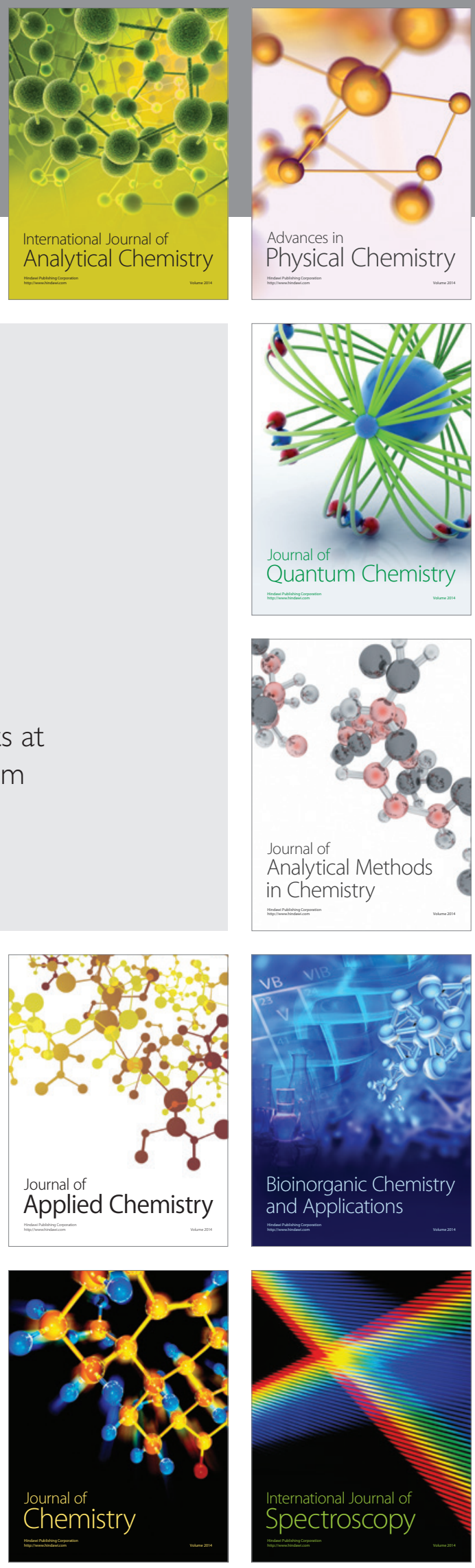